\title{
On monotonic and logarithmic concavity properties of generalized $k$-Bessel function
}

\author{
İbrahim Aktaş(1) \\ Department of Mathematics, Kamil Özdă̆ Science Faculty, Karamanoğlu Mehmetbey University, Yunus \\ Emre Campus, 70100, Karaman, Turkey
}

\begin{abstract}
In this study, our main objective is to determine some monotonic and log-concavity properties of generalized $k$-Bessel function by using its Hadamard product representation and some earlier results on power series. In addition, by using the relationships between Besseltype special functions and some basic functions, we present some specific examples related to the monotonic and log-concavity properties of some trigonometric and hyperbolic functions.
\end{abstract}

Mathematics Subject Classification (2020). 33E50

Keywords. $k$-Gamma functions, $k$-Bessel function, monotonicity, log-concavity

\section{Introduction and preliminaries}

In the recent years many geometric and monotonic properties of some special functions like Bessel, Struve, Lommel, Mittag-Leffler, Wright and their generalizations were investigated by many authors. Comprehensive information about these investigations can be found in $[1-8,10,14]$ and references therein. Especially, some inequalities and monotonic properties of the above mentioned functions are usefull in engineering, physics, probability and statistics, and economics. It is known that log-concavity and log-convexity properties have a crucial role in economics. Comprehensive information about the log-concavity and the log-convexity properties can be found in [13] and its references. In this study, motivated by the some earlier results which are given in $[14,15]$, our main aim is to present some monotonic and log-concavity properties of generalized $k$-Bessel functions. Moreover, we give some specific examples regarding our obtained result by using the relationships between Bessel-type functions and elementary trigonometric and hyperbolic functions.

It is known that, most of special functions can be defined with the help of Euler's gamma function. Therefore, we would like to remind the definitions of gamma function and its $k$-generalization. The Euler's gamma function $\Gamma$ is defined by the following improper integral, for $x>0$ :

$$
\Gamma(x)=\int_{0}^{\infty} t^{x-1} e^{-t} d t .
$$

Email address: aktasibrahim38@gmail.com; ibrahimaktas@kmu.edu.tr Received: 17.09.2019; Accepted: 28.05.2020 
Also, the $k$-gamma function is defined by (see [12])

$$
\Gamma_{k}(x)=\int_{0}^{\infty} t^{x-1} e^{\frac{-t^{k}}{k}} d t
$$

for $k>0$. We know that the $k$-gamma function $\Gamma_{k}$ reduces to the classical gamma function $\Gamma$ when $k \rightarrow 1$. In addition, Pochammer $k$-symbol is defined by

$$
(\lambda)_{n, k}=\lambda(\lambda+k)(\lambda+2 k) \ldots((\lambda+(n-1) k))
$$

for $\lambda \in \mathbb{C}, k \in \mathbb{R}$ and $n \in \mathbb{N}^{+}$. Other properties of Pochammer $k$-symbol and $k$-gamma function can be found in [12].

In this paper, we are considering the generalized $k$-Bessel function defined by the following series representation (see [14]):

$$
W_{\nu, c}^{k}(x)=\sum_{n=0}^{\infty} \frac{(-c)^{n}}{n ! \Gamma_{k}(n k+\nu+k)}\left(\frac{x}{2}\right)^{2 n+\frac{\nu}{k}}
$$

for $k>0, \nu>-1$ and $c \in \mathbb{R}$. It is clear that the generalized $k$-Bessel function reduces to classical Bessel and modified Bessel functions for appropriate values of the parameters $k$ and $c$, respectively. More precisely, taking $k=c=1$ and $k=-c=1$ in (1.1), we have that

and

$$
W_{\nu, 1}^{1}(x)=\sum_{n=0}^{\infty} \frac{(-1)^{n}}{n ! \Gamma(n+\nu+1)}\left(\frac{x}{2}\right)^{2 n+\nu}=J_{\nu}(x)
$$

$$
W_{\nu,-1}^{1}(x)=\sum_{n=0}^{\infty} \frac{1}{n ! \Gamma(n+\nu+1)}\left(\frac{x}{2}\right)^{2 n+\nu}=I_{\nu}(x),
$$

where $J_{\nu}(x)$ and $I_{\nu}(x)$ denote classical Bessel and modified Bessel functions of the first kind, respectively. In [15], the author studied some geomertric properties such as radii of starlikeness and convexity of generalized $k$-Bessel function. Also, the author gave an infinite product representation of generalized $k$-Bessel function by using Hadamard's theorem as follow (see [15, Lemma 1.1]):

$$
W_{\nu, c}^{k}(x)=\frac{\left(\frac{x}{2}\right)^{\frac{\nu}{k}}}{\Gamma_{k}(\nu+k)} \prod_{n \geq 1}\left(1-\frac{x^{2}}{k w_{\nu, c, n}^{2}}\right),
$$

where ${ }_{k} w_{\nu, c, n}$ denotes $n$th positive zero of generalized $k$-Bessel function $W_{\nu, c}^{k}(x)$.

Now, we would like to give the definition of logarithmic concavity of a function.

Definition 1.1 ([13]). A function $f$ is said to be log-concave on interval $(a, b)$ if the function $\log f$ is a concave function on $(a, b)$.

To show log-concavity of a function $f$ on the interval $(a, b)$, it is sufficient to show one of the following two conditions:

i. $\frac{f^{\prime}}{f}$ monotone decreasing on $(a, b)$.

ii. $\log f^{\prime \prime}<0$.

Also the following lemma due to Biernacki and Krzyż (see [11]) will be used in order to prove some monotonic properties of the mentioned functions.

Lemma 1.2. Consider the power series $f(x)=\sum_{n \geq 0} a_{n} x^{n}$ and $g(x)=\sum_{n \geq 0} b_{n} x^{n}$, where $a_{n} \in \mathbb{R}$ and $b_{n}>0$ for all $n \in\{0,1, \ldots\}$, and suppose that both converge on $(-r, r), r>0$. If the sequence $\left\{\frac{a_{n}}{b_{n}}\right\}_{n \geq 0}$ is increasing(decreasing), then the function $x \mapsto\left(\frac{f(x)}{g(x)}\right)$ is also increasing (decreasing) on $(0, r)$.

It is important to note that the above result remains true for the even or odd functions.

The outcomes of our paper is as follow: In Section 2, we give our main results and their consequences, while the Section 3 is devoted for some applications of our main results. 


\section{Main results}

In this section, we present our main results and their consequences.

Theorem 2.1. Let $k>0, k+\nu>0, c \in \mathbb{R}$ and ${ }_{k} w_{\nu, c, n}$ denote the nth positive zero of the generalized $k$-Bessel function $W_{\nu, c}^{k}(x)$. Further, consider the following sets:

$$
\delta_{1}=\bigcup_{n \geq 1}\left({ }_{k} w_{\nu, c, 2 n-1},{ }_{k} w_{\nu, c, 2 n}\right), \delta_{2}=\bigcup_{n \geq 1}\left({ }_{k} w_{\nu, c, 2 n},{ }_{k} w_{\nu, c, 2 n+1}\right) \text { and } \delta_{3}=\left[0,{ }_{k} w_{\nu, c, 1}\right) \cup \delta_{2} .
$$

The generalized $k$-Bessel function

$$
\Theta_{\nu, c}^{k}(x)=\Gamma_{k}(\nu+k) 2^{\frac{\nu}{k}} x^{-\frac{\nu}{k}} W_{\nu, c}^{k}(x)=\sum_{n=0}^{\infty} \frac{(-c)^{n}}{n !(\nu+k)_{n, k}}\left(\frac{x}{2}\right)^{2 n}
$$

has the following properties:

a. the function $x \mapsto \Theta_{\nu, c}^{k}(x)$ is negative on $\delta_{1}$ and it is positive on $\delta_{3}$,

b. the function $x \mapsto \Theta_{\nu, c}^{k}(x)$ is a decreasing function on $\left[0,{ }_{k} w_{\nu, c, 1}\right)$,

c. the function $x \mapsto \Theta_{\nu, c}^{k}(x)$ is strictly log-concave on $\delta_{3}$.

Proof. a. If we consider the infinite product representation of generalized $k$-Bessel function $W_{\nu, c}^{k}(x)$ which is given by (1.4), then it can be easily seen that the function $\Theta_{\nu, c}^{k}(x)$ can be written by the following product representation:

$$
\Theta_{\nu, c}^{k}(x)=\prod_{n \geq 1}\left(1-\frac{x^{2}}{{ }_{k} w_{\nu, c, n}^{2}}\right) .
$$

In order to investigate the sign of the function $x \mapsto \Theta_{\nu, c}^{k}(x)$ on the mentioned sets, we rewrite the function $x \mapsto \Theta_{\nu, c}^{k}(x)$ as

$$
\Theta_{\nu, c}^{k}(x)=U_{n} V_{n}
$$

where

$$
U_{n}=\prod_{n \geq 1} \frac{{ }_{k} w_{\nu, c, n}+x}{{ }_{k} w_{\nu, c, n}^{2}} \text { and } V_{n}=\prod_{n \geq 1}\left({ }_{k} w_{\nu, c, n}-x\right) .
$$

It is clear that $U_{n}>0$ for all $x \in \mathbb{R}^{+} \cup\{0\}$. On the other hand, since

$$
0<{ }_{k} w_{\nu, c, 1}<{ }_{k} w_{\nu, c, 2}<\cdots<{ }_{k} w_{\nu, c, n}<\cdots,
$$

we can say that, if $x \in\left({ }_{k} w_{\nu, c, 2 n-1},{ }_{k} w_{\nu, c, 2 n}\right)$, then the first $(2 n-1)$ terms of $V_{n}$ are strictly negative and remained terms are strictly positive. Also, if $x \in\left({ }_{k} w_{\nu, c, 2 n},{ }_{k} w_{\nu, c, 2 n+1}\right)$, then the first $2 n$ terms of $V_{n}$ are strictly negative and the rest is strictly positive. In addition, all the terms of $V_{n}$ are strictly positive for $x \in\left[0,{ }_{k} w_{\nu, c, 1}\right)$. As a consequence, the function $x \mapsto \Theta_{\nu, c}^{k}(x)$ is negative on $\delta_{1}$ and it is positive on $\delta_{3}$.

b. We know from part $\boldsymbol{a}$. that the function $x \mapsto \Theta_{\nu, c}^{k}(x)$ is positive on the interval $\left[0,{ }_{k} w_{\nu, c, 1}\right)$. The logarithmic differentation of (2.2) implies that

$$
\frac{\left(\Theta_{\nu, c}^{k}(x)\right)^{\prime}}{\Theta_{\nu, c}^{k}(x)}=\sum_{n=1}^{\infty} \frac{2 x}{x^{2}-{ }_{k} w_{\nu, c, n}^{2}} .
$$

Thus, we get

$$
\left(\Theta_{\nu, c}^{k}(x)\right)^{\prime}=\Theta_{\nu, c}^{k}(x) \sum_{n=1}^{\infty} \frac{2 x}{x^{2}-{ }_{k} w_{\nu, c, n}^{2}}<0
$$

for all $x \in\left[0,{ }_{k} w_{\nu, c, 1}\right)$. As a result, the function $x \mapsto \Theta_{\nu, c}^{k}(x)$ is a decreasing function on $\left[0,{ }_{k} w_{\nu, c, 1}\right)$. 
c. In order to prove log-concavity of the function $x \mapsto \Theta_{\nu, c}^{k}(x)$, we need to show that

$$
\frac{d^{2}}{d x^{2}}\left[\log \Theta_{\nu, c}^{k}(x)\right]<0
$$

for all $x \in \delta_{3}$. Now, by using the infinite product representation of the function $\Theta_{\nu, c}^{k}(x)$ which is given by $(2.2)$ we infer that

$$
\begin{aligned}
\frac{d^{2}}{d x^{2}}\left[\log \Theta_{\nu, c}^{k}(x)\right] & =\frac{d^{2}}{d x^{2}}\left[\log \prod_{n \geq 1}\left(1-\frac{x^{2}}{k w_{\nu, c, n}^{2}}\right)\right] \\
& =\frac{d}{d x}\left[\frac{d}{d x} \sum_{n=1}^{\infty} \log \left(1-\frac{x^{2}}{{ }_{k} w_{\nu, c, n}^{2}}\right)\right] \\
& =\frac{d}{d x} \sum_{n=1}^{\infty} \frac{-2 x}{k w_{\nu, c, n}^{2}-x^{2}} \\
& =-2 \sum_{n=1}^{\infty} \frac{{ }_{k} w_{\nu, c, n}^{2}+x^{2}}{\left({ }_{k} w_{\nu, c, n}^{2}-x^{2}\right)^{2}} \\
& <0
\end{aligned}
$$

for $x \in \delta_{3}$. Thus, the proof is completed.

By setting $k=c=1$ and $k=1, c=-1$ in the Theorem 2.1 we have the following properties for the classical Bessel and modified Bessel functions, respectively.

Corollary 2.2. Let $\nu>-1$ and $j_{\nu, n}$ denote the nth positive zero of the classical Bessel function $J_{\nu}(x)$. Further, consider the next sets:

$$
A_{1}=\bigcup_{n \geq 1}\left(j_{\nu, 2 n-1}, j_{\nu, 2 n}\right), A_{2}=\bigcup_{n \geq 1}\left(j_{\nu, 2 n}, j_{\nu, 2 n+1}\right) \text { and } A_{3}=\left[0, j_{\nu, 1}\right) \cup A_{2} .
$$

The following assertions are true:

a. the function $\Theta_{\nu, 1}^{1}(x)=\Gamma(\nu+1) 2^{\nu} x^{-\nu} J_{\nu}(x)$ is negative on $A_{1}$ and it is positive on $A_{3}$,

b. the function $\Theta_{\nu, 1}^{1}(x)=\Gamma(\nu+1) 2^{\nu} x^{-\nu} J_{\nu}(x)$ is a decreasing function on $\left[0, j_{\nu, 1}\right)$,

c. the function $\Theta_{\nu, 1}^{1}(x)=\Gamma(\nu+1) 2^{\nu} x^{-\nu} J_{\nu}(x)$ is strictly log-concave on $A_{3}$.

Corollary 2.3. Let $\nu>-1$ and $\epsilon_{\nu, n}$ denote the $n$th positive zero of the modified Bessel function $I_{\nu}(x)$. Further, consider the next sets:

$$
B_{1}=\bigcup_{n \geq 1}\left(\epsilon_{\nu, 2 n-1}, \epsilon_{\nu, 2 n}\right), B_{2}=\bigcup_{n \geq 1}\left(\epsilon_{\nu, 2 n}, \epsilon_{\nu, 2 n+1}\right) \text { and } B_{3}=\left[0, \epsilon_{\nu, 1}\right) \cup B_{2} .
$$

The following assertions are true:

a. the function $\Theta_{\nu,-1}^{1}(x)=\Gamma(\nu+1) 2^{\nu} x^{-\nu} I_{\nu}(x)$ is negative on $B_{1}$ and it is positive on $B_{3}$,

b. the function $\Theta_{\nu,-1}^{1}(x)=\Gamma(\nu+1) 2^{\nu} x^{-\nu} I_{\nu}(x)$ is a decreasing function on $\left[0, \epsilon_{\nu, 1}\right)$,

c. the function $\Theta_{\nu,-1}^{1}(x)=\Gamma(\nu+1) 2^{\nu} x^{-\nu} I_{\nu}(x)$ is strictly log-concave on $B_{3}$.

Theorem 2.4. Let $k>0, \nu>0, c \in \mathbb{R}$ and ${ }_{k} w_{\nu, c, n}$ denote the nth positive zero of the generalized $k$-Bessel function $W_{\nu, c}^{k}(x)$. Then, the function $x \mapsto W_{\nu, c}^{k}(x)$ is strictly logconcave on $\left(0,{ }_{k} w_{\nu, c, 1}\right) \cup \delta_{2}$.

Proof. It is known that the product of two strictly log-concave function is also strictly log-concave. By using this fact it is possible to prove the log-concavity of the generalized 
$k$-Bessel function $W_{\nu, c}^{k}(x)$ on $\delta_{3}$. Hence, we rewrite the function $W_{\nu, c}^{k}(x)$ as follow:

$$
W_{\nu, c}^{k}(x)=\frac{\left(\frac{x}{2}\right)^{\frac{\nu}{k}}}{\Gamma_{k}(\nu+k)} \Theta_{\nu, c}^{k}(x) .
$$

Since

$$
\frac{d^{2}}{d x^{2}}\left[\log \left(\frac{x}{2}\right)^{\frac{\nu}{k}}\right]=-\frac{\nu}{k x^{2}}<0
$$

for $\nu>0, k>0$ and $x \in \mathbb{R}^{+}$, the function $x \mapsto\left(\frac{x}{2}\right)^{\frac{\nu}{k}}$ is strictly log-concave on $\mathbb{R}^{+}$. In addition, it is known from part $\boldsymbol{c}$. of Theorem 2.1 that the function $\Theta_{\nu, c}^{k}(x)$ is strictly logconcave on $\delta_{3}$. As a result, the function $W_{\nu, c}^{k}(x)$ is strictly log-concave on $\left(0,{ }_{k} w_{\nu, c, 1}\right) \cup \delta_{2}$ as a product of two strictly log-concave functions.

Now, by taking $k=c=1$ and $k=1, c=-1$ in Theorem 2.4, we deduce the following properties for the classical Bessel and modified Bessel functions, respectively.

Corollary 2.5. The function $x \mapsto J_{\nu}(x)$ is strictly log-concave on $\left(0, j_{\nu, 1}\right) \cup A_{2}$, while the function $x \mapsto I_{\nu}(x)$ is strictly log-concave on $\left(0, \epsilon_{\nu, 1}\right) \cup B_{2}$.

Our last main result is the following theorem.

Theorem 2.6. The function $\Phi_{\nu,-1}^{k}(x)=\frac{x\left(\Theta_{\nu,-1}^{k}(x)\right)^{\prime}}{\Theta_{\nu,-1}^{k}(x)}$ is increasing on $(0, \infty)$ for $v>-1$ and $\nu+k>0$.

Proof. If we put $c=-1$ in definition of the function $\Theta_{\nu, c}^{k}(x)$, then we get the following infinite series representation for the function $\Theta_{\nu,-1}^{k}(x)$, that is,

$$
\Theta_{\nu,-1}^{k}(x)=\sum_{n=0}^{\infty} \mathcal{P}_{n, \nu, k} x^{2 n}
$$

where $\mathcal{P}_{n, \nu, k}=\frac{1}{n ! 4^{n}(\nu+k)_{n, k}}$. Differentiating both sides of the equality $(2.3)$ and by multiplying by $x$ obtained equality, we get that

$$
x\left(\Theta_{\nu,-1}^{k}(x)\right)^{\prime}=\sum_{n=0}^{\infty} \mathcal{R}_{n, \nu, k} x^{2 n},
$$

where $\mathcal{R}_{n, \nu, k}=\frac{2 n}{n ! 4^{n}(\nu+k)_{n, k}}$. According to Cauchy-Hadamard theorem for power series, it can be easily shown that both power series $\sum_{n=0}^{\infty} \mathcal{P}_{n, \nu, k} x^{2 n}$ and $\sum_{n=0}^{\infty} \mathcal{R}_{n, \nu, k} x^{2 n}$ are convergent on $(-\infty, \infty)$, since

$$
\lim _{n \rightarrow \infty}\left|\frac{\mathcal{P}_{n, \nu, k}}{\mathcal{P}_{n+1, \nu, k}}\right|=\lim _{n \rightarrow \infty}\left|\frac{\mathcal{R}_{n, \nu, k}}{\mathcal{R}_{n+1, \nu, k}}\right|=\infty .
$$

Here we used the equality $(\nu+k)_{n+1, k}=(\nu+k+n k)(\nu+k)_{n, k}$ for the Pochammer $k$ symbol. On the other hand, it can be easily seen that $\mathcal{R}_{n, \nu, k} \in \mathbb{R}$ and $\mathcal{P}_{n, \nu, k}>0$ for all $n \in\{0,1, \ldots\}, \nu>-1$ and $\nu+k>0$. Now, if we consider the sequence

$$
U_{n}=\frac{\mathcal{R}_{n, \nu, k}}{\mathcal{P}_{n, \nu, k}}=2 n,
$$

then we have

$$
\frac{U_{n+1}}{U_{n}}=\frac{n+1}{n}>1 .
$$

So the sequence $\left\{U_{n}\right\}_{n \geq 0}$ is increasing. The proof is completed by applying Lemma 1.2. 


\section{Applications}

In this section, we want to give some applications of our main results. Therefore, we consider the relationships among of the functions $x \mapsto \Theta_{\nu, c}^{k}(x), x \mapsto J_{\nu}(x)$ and $x \mapsto I_{\nu}(x)$. We know from (1.2) and (1.3) that, the following equalities

$$
W_{\nu, 1}^{1}(x)=J_{\nu}(x) \text { and } W_{\nu,-1}^{1}(x)=I_{\nu}(x)
$$

hold true for $k=c=1$ and $k=1, c=-1$, respectively. On the other hand, we know from [9] that some basic trigonometric and hyperbolic functions can be written in terms of Bessel and modified Bessel functions for some special values of $\nu$. Especially, for $\nu=-\frac{1}{2}, \nu=\frac{1}{2}$ and $\nu=\frac{3}{2}$ we have the following basic trigonometric and hyperbolic functions:

$$
J_{-\frac{1}{2}}(x)=\sqrt{\frac{2}{\pi x}} \cos x, \quad J_{\frac{1}{2}}(x)=\sqrt{\frac{2}{\pi x}} \sin x, \quad J_{\frac{3}{2}}(x)=\sqrt{\frac{2}{\pi x}}\left(\frac{\sin x}{x}-\cos x\right)
$$

and

$$
I_{-\frac{1}{2}}(x)=\sqrt{\frac{2}{\pi x}} \cosh x, \quad I_{\frac{1}{2}}(x)=\sqrt{\frac{2}{\pi x}} \sinh x, \quad I_{\frac{3}{2}}(x)=-\sqrt{\frac{2}{\pi x}}\left(\frac{\sinh x}{x}-\cosh x\right) .
$$

By using above relationships, we have the followings:

$$
\Theta_{-\frac{1}{2}, 1}^{1}(x)=\cos x, \quad \Theta_{\frac{1}{2}, 1}^{1}(x)=\frac{\sin x}{x}, \quad \Theta_{\frac{3}{2}, 1}^{1}(x)=3\left(\frac{\sin x-x \cos x}{x^{3}}\right)
$$

and

$$
\Theta_{-\frac{1}{2},-1}^{1}(x)=\cosh x, \quad \Theta_{\frac{1}{2},-1}^{1}(x)=\frac{\sinh x}{x}, \quad \Theta_{\frac{3}{2},-1}^{1}(x)=3\left(\frac{x \cosh x-\sinh x}{x^{3}}\right)
$$

respectively.

Now, by using the above relationships in Corollary 2.2, Corollary 2.3, Corollary 2.5 and Theorem 2.6, respectively, we can give the following some interesting examples.

Example 3.1. The following assertions hold true.

i. The function $x \mapsto \Theta_{-\frac{1}{2}, 1}^{1}(x)=\cos x$ is strictly log-concave on $\left[0, j_{-\frac{1}{2}, 1}\right) \cup T_{1}$, where $T_{1}=\bigcup_{n \geq 1}\left(j_{-\frac{1}{2}, 2 n}, j_{-\frac{1}{2}, 2 n+1}\right)$ and $j_{-\frac{1}{2}, n}$ denotes the $n$th positive zero of the equation $\cos x=0$.

ii. The function $x \mapsto \Theta_{\frac{1}{2}, 1}^{1}(x)=\frac{\sin x}{x}$ is strictly log-concave on $\left[0, j_{\frac{1}{2}, 1}\right) \cup T_{2}$, where $T_{2}=\bigcup_{n \geq 1}\left(j_{\frac{1}{2}, 2 n}, j_{\frac{1}{2}, 2 n+1}\right)$ and $j_{\frac{1}{2}, n}$ denotes the $n$th positive zero of the equation $\sin x=0$.

iii. The function $x \mapsto \Theta_{\frac{3}{2}, 1}^{1}(x)=3\left(\frac{\sin x-x \cos x}{x^{3}}\right)$ is strictly log-concave on $\left[0, j_{\frac{3}{2}, 1}\right) \cup T_{3}$, where $T_{3}=\bigcup_{n \geq 1}\left(j_{\frac{3}{2}, 2 n}, j_{\frac{3}{2}, 2 n+1}\right)$ and $j_{\frac{3}{2}, n}$ denotes the $n$th positive zero of the equation $\tan x=x$.

Example 3.2. The following statements are valid.

i. The function $x \mapsto \Theta_{-\frac{1}{2},-1}^{1}(x)=\cosh x$ is strictly log-concave on $\left[0, \epsilon_{-\frac{1}{2}, 1}\right) \cup S_{1}$, where $S_{1}=\bigcup_{n \geq 1}\left(\epsilon_{-\frac{1}{2}, 2 n}, \epsilon_{-\frac{1}{2}, 2 n+1}\right)$ and $\epsilon_{-\frac{1}{2}, n}$ denotes the $n$th positive zero of the equation $\cosh x=0$.

ii. The function $x \mapsto \Theta_{\frac{1}{2},-1}^{1}(x)=\frac{\sinh x}{x}$ is strictly log-concave on $\left[0, \epsilon_{\frac{1}{2}, 1}\right) \cup S_{2}$, where $S_{2}=\bigcup_{n \geq 1}\left(\epsilon_{\frac{1}{2}, 2 n}, \epsilon_{\frac{1}{2}, 2 n+1}\right)$ and $\epsilon_{\frac{1}{2}, n}$ denotes the $n$th positive zero of the equation $\sinh x=0$. 
iii. The function $x \mapsto \Theta_{\frac{3}{2},-1}^{1}(x)=3\left(\frac{\sinh x-x \cosh x}{x^{3}}\right)$ is strictly log-concave on $\left[0, \epsilon_{\frac{3}{2}, 1}\right) \cup$ $S_{3}$, where $S_{3}=\bigcup_{n \geq 1}\left(\epsilon_{\frac{3}{2}, 2 n}, \epsilon_{\frac{3}{2}, 2 n+1}\right)$ and $\epsilon_{\frac{3}{2}, n}$ denotes the $n$th positive zero of the equation $\tanh x=x$.

Example 3.3. The following assertions hold true.

i. The function $J_{-\frac{1}{2}}(x)=\sqrt{\frac{2}{\pi x}} \cos x$ is strictly log-concave on $\left[0, j_{-\frac{1}{2}, 1}\right) \cup T_{1}$.

ii. The function $J_{\frac{1}{2}}(x)=\sqrt{\frac{2}{\pi x}} \sin x$ is strictly log-concave on $\left[0, j_{\frac{1}{2}, 1}\right) \cup T_{2}$.

iii. The function $J_{\frac{3}{2}}(x)=\sqrt{\frac{2}{\pi x}}\left(\frac{\sin x}{x}-\cos x\right)$ is strictly log-concave on $\left[0, j_{\frac{3}{2}, 1}\right) \cup T_{3}$.

iv. The function $I_{-\frac{1}{2}}(x)=\sqrt{\frac{2}{\pi x}} \cosh x$ is strictly log-concave on $\left[0, \epsilon_{-\frac{1}{2}, 1}\right) \cup S_{1}$.

v. The function $I_{\frac{1}{2}}(x)=\sqrt{\frac{2}{\pi x}} \sinh x$ is strictly log-concave on $\left[0, \epsilon_{\frac{1}{2}, 1}\right) \cup S_{2}$.

vi. The function $I_{\frac{3}{2}}(x)=-\sqrt{\frac{2}{\pi x}}\left(\frac{\sinh x}{x}-\cosh x\right)$ is strictly log-concave on $\left[0, \epsilon_{\frac{3}{2}, 1}\right) \cup$ $S_{3}$.

Example 3.4. The following functions

$$
\Phi_{-\frac{1}{2},-1}^{1}(x)=x \tanh x, \quad \Phi_{\frac{1}{2},-1}^{1}(x)=x \operatorname{coth} x-1
$$

and

are increasing functions on $(0, \infty)$.

$$
\Phi_{\frac{3}{2},-1}^{1}(x)=\frac{\left(x^{2}+3\right) \sinh x-3 x \cosh x}{x \cosh x-\sinh x}
$$

\section{References}

[1] İ. Aktaş, On some properties of hyper-Bessel and related functions, TWMS J. App. and Eng. Math. 9 (1), 30-37, 2019.

[2] İ. Aktaş, Partial sums of Hyper-Bessel function with applications, Hacet. J. Math. Stat. 49 (1), 380-388, 2020.

[3] İ. Aktaş and Á. Baricz, Bounds for the radii of starlikeness of some q-Bessel functions, Results Math. 72 (1-2), 947-963, 2017.

[4] İ. Aktaş and H. Orhan, Bounds for the radii of convexity of some q-Bessel functions, Bull. Korean Math. Soc. 57 (2), 355-369, 2020.

[5] İ. Aktaş, Á. Baricz and H. Orhan, Bounds for the radii of starlikeness and convexity of some special functions, Turkish J. Math. 42 (1), 211-226, 2018.

[6] İ. Aktaş, Á. Baricz and S. Singh, Geometric and monotonic properties of hyper-Bessel functions, Ramanujan J. 51 (2), 275-295, 2020.

[7] İ. Aktaş, Á. Baricz and N. Yağmur, Bounds for the radii of univalence of some special functions, Math. Inequal. Appl. 20 (3), 825-843, 2017.

[8] Á. Baricz, Geometric properties of generalized Bessel functions, Publ. Math. Debrecen $73(1-2), 155-178,2008$.

[9] Á. Baricz, Generalized Bessel Functions of the First Kind, Lecture Notes in Mathematics, Springer-Verlag, 2010.

[10] Á. Baricz and T.K. Pogány, Functional inequalities of modified Struve functions, Proc. Roy. Soc. Edinburgh Sect. A, 144 (5), 891-904, 2014.

[11] M. Biernacki and J. Krzyż, On the monotonity of certain functionals in the theory of analytic functions, Ann. Univ. Mariae Curie-Skłodowska Sect. A, 9, 135-147, 1955.

[12] R. Díaz and E. Pariguan, On hypergeometric functions and Pochhammer k-symbol, Divulgaciones Mathemáticas, 15 (2), 179-192, 2007.

[13] G.R. Mohtasami Borzadaran and H.A. Mohtasami Borzadaran, Log-concavity property for some well-known distributions, Surv. Math. Appl. 6, 203-219, 2011. 
[14] S.R. Mondal and M.S. Akel, Differential equation and inequalities of the generalized $k$-Bessel functions, J. Inequal. Appl., 2018:175. doi: 10.1186/s13660-018-1772-1

[15] E. Toklu, Radii of starlikeness and convexity of generalized $k$-Bessel functions, arXiv:1902.09979, 2019. 